\title{
3-Hydroxyacyl-CoA Dehydrogenase
}

National Cancer Institute

\section{Source}

National Cancer Institute. 3-Hydroxyacyl-CoA Dehydrogenase. NCI Thesaurus. Code C112050.

A family of enzymes that oxidize (S)-3-hydroxyacyl-CoA and NAD+ and produce 3oxoacyl-CoA, $\mathrm{NADH}$, and $\mathrm{H}+$. These proteins are involved in the metabolism of both fatty acids and amino acids. 